\title{
EXERCÍCIOS DURANTE A QUIMIOTERAPIA: revisão bibliográfica
}

\author{
Rebeca Garcia Rosa FERREIRA ${ }^{1}$
}

Laura Ferreira de Rezende FRANCO ${ }^{2}$

\begin{abstract}
${ }^{1}$ Fisioterapeuta. Mestre em Desenvolvimento Sustentável e Qualidade de Vida pelo Centro Universitário das Faculdades Associadas de Ensino de São João da Boa Vista (UNIFAE), Docente do curso de Fisioterapia do Centro Universitário das Faculdades Associadas de Ensino de São João da Boa Vista (UNIFAE). rebeca@fae.br

${ }^{2}$ Fisioterapeuta. Mestre e Doutora em Tocoginecologia pela Universidade Estadual de Campinas (Unicamp). PósDoutora pelo Departamento de Obstetrícia, Ginecologia e Mastologia pela Universidade Estadual Paulista (UNESP). Docente do Curso de Fisioterapia e Docente do Curso de Mestrado em Desenvolvimento Sustentável e Qualidade de Vida do Centro Universitário das Faculdades de Associadas de Ensino de São João da Boa Vista (UNIFAE). laura@fae.br
\end{abstract}

\section{Recebido em: 09/05/2016 - Aprovado em: 18/09/2016 - Disponibilizado em: 18/12/2016}

\section{RESUMO:}

Os exercícios tem sido sugeridos durante o tratamento quimioterápico no pós-operatório de câncer de mama para minimizar diversos efeitos colaterais. O objetivo foi realizar uma revisão da literatura sobre os efeitos dos exercícios físicos durante a quimioterapia no tratamento de câncer de mama. O método da pesquisa foi realizada nas bases de dados eletrônicas, MEDLINE, PubMed e SciELO, através de descritores: "câncer de mama", "qualidade de vida", "exercícios físicos", "quimioterapia", "mielodepressão" e "fadiga muscular". Foi realizada a busca por ensaios clínicos randomizados em português, inglês e espanhol, publicados entre 1997 à 2015. Nos resultados os 14 artigos selecionados demonstraram que exercícios de força muscular e aeróbicos melhoram significativamente a mielodepressão, fadiga muscular e qualidade de vida causados pela quimioterapia. Na conclusão verificou-se a importância dos exercícios físicos durante o tratamento quimioterápico no câncer de mama.

Palavras-chave: Câncer de Mama. Qualidade de Vida. Mielodepressão. Fadiga Muscular. Exercício Físico.

\begin{abstract}
:
The exercises have been suggested during chemotherapy in breast cancer after surgery to minimize various side effects. The objective was to review the literature on the effects of exercise during chemotherapy in the treatment of breast cancer. The method of research was carried out in electronic databases MEDLINE, PubMed and SciELO by descriptors: "breast cancer", "quality of life", "exercise", "chemotherapy", "myelosuppression" and "muscle fatigue ". It performed a search for randomized clinical trials in Portuguese, English and Spanish, published between 1997 to 2015. In the 14 articles selected results showed that muscle strength and aerobic exercise significantly improve myelosuppression, muscle fatigue and quality of life caused by chemotherapy. In conclusion there was the importance of exercise during chemotherapy in breast cancer.
\end{abstract}

Keywords: Breast Cancer. Quality of life. Myelosuppression. Muscle fatigue. Physical exercise.

\section{INTRODUÇÃO}

O câncer de mama é considerado uma doença sistêmica, portanto a quimioterapia é recomendada em praticamente todos os casos (BRASIL, 2015). O principal efeito colateral da quimioterapia é a mielodepressão ou mielotoxicidade, podendo proporcionar $\mathrm{o}$ aparecimento de choque séptico, infecções, anemia, risco de hemorragia e morte. A morte é causada devido a infecções, decorrentes da neutropenia (SCHWENKGLENKS, 2006).

A fadiga também é conhecida como um efeito colateral importante, sendo que 40 a $100 \%$ dos pacientes relatam ter experimentado esse sintoma (RICHARDSON et al, 1998), reduzindo a capacidade funcional e prejudicando a habilidade do paciente em interagir com a família e amigos (SKALA et 
al, 1992). Apresenta-se como uma sensação persistente e subjetiva de cansaço relacionado ao câncer ou tratamento do câncer que interfere na funcionalidade (MOCK et al, 2005).

Muitos pacientes que apresentam mielodepressão e/ou fadiga acabam diminuindo suas atividades físicas decorrentes dos efeitos colaterais do tratamento quimioterápico, permitindo o imobilismo, inabilidade física e fadiga muscular propiciando dessa forma complicações como a susceptibilidade de infecções podendo levar a hospitalizações ou até mesmo óbito.

Dessa forma a proposta desse artigo foi fazer uma revisão dos efeitos do exercício físico na mielodepressão e fadiga muscular durante a quimioterapia adjuvante no câncer de mama.

\section{MÉTODOS}

A pesquisa foi realizada nas bases de dados eletrônicas, MEDLINE, PubMed e SciELO, através de descritores: "câncer de mama", "qualidade de vida", "exercícios físicos", "quimioterapia", "mielodepressão" e "fadiga muscular". Foi realizada a busca por ensaios clínicos randomizados em português , inglês e espanhol, publicados entre 1997 à 2015.

\section{RESULTADOS}

Os efeitos dos exercícios no câncer de mama em pacientes submetidos à quimioterapia e os métodos de avaliação utilizados pelos artigos estão descritos na Tabela 1.

Tabela 1. Efeitos dos exercícios no câncer de mama

\begin{tabular}{|c|c|c|}
\hline $\begin{array}{lr}\text { Autor } & \text { e } \\
\text { ano } & \text { de } \\
\text { publicação }\end{array}$ & $\begin{array}{l}\text { Caracterização } \\
\text { da amostra }\end{array}$ & $\begin{array}{l}\text { *Intervenções } \\
* * \text { Resultados }\end{array}$ \\
\hline $\begin{array}{l}\text { Mock et al, } \\
1997\end{array}$ & $\begin{array}{l}46 \text { mulheres } \\
\text { com fadiga } \\
\text { muscular, } \\
\text { ansiedade } \text { e } \\
\text { insônia no } \\
\text { câncer de } \\
\text { mama }\end{array}$ & $\begin{array}{l}\text { *Programa de } \\
\text { caminhada. } \\
* * \text { Melhora } \\
\text { funcionamento } \\
\text { físico, ansiedade, } \\
\text { fadiga muscular e } \\
\text { em relação ao sono. }\end{array}$ \\
\hline $\begin{array}{l}\text { Mock et al, } \\
2001\end{array}$ & $\begin{array}{l}52 \text { mulheres } \\
\text { com fadiga } \\
\text { muscular no } \\
\text { câncer } \\
\text { mama }\end{array}$ & $\begin{array}{l}\text { *Exercícios } \\
\text { (caminhada em casa) } \\
\text { Exercícios } \\
\text { (bicicletas } \\
\text { laboratórios } \\
\text { hospital). } \\
\text { **Diminuição dos } \\
\text { níveis de fadiga } \\
\text { muscular. }\end{array}$ \\
\hline $\begin{array}{l}\text { Courneya } \\
\text { et al, } 2003\end{array}$ & $\begin{array}{l}53 \text { mulheres } \\
\text { com câncer de } \\
\text { mama }\end{array}$ & $\begin{array}{l}\text { *Exercício aeróbico } \\
3 \text { vezes por semana } \\
\text { durante } 15 \text { semanas. } \\
\text { **Melhora da } \\
\text { qualidade de vida, } \\
\text { melhora no pico de } \\
\text { consumo de } \\
\text { oxigênio. }\end{array}$ \\
\hline $\begin{array}{l}\text { Thorsen et } \\
\text { al, } 2005\end{array}$ & $\begin{array}{l}111 \text { mulheres } \\
\text { com câncer de } \\
\text { mama }\end{array}$ & 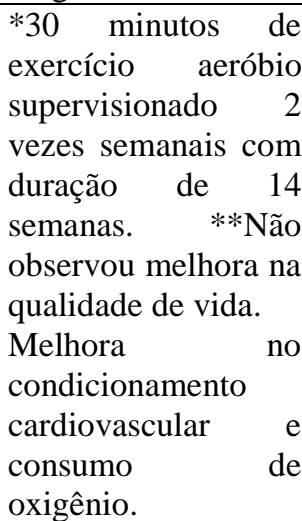 \\
\hline $\begin{array}{l}\text { Mock et al, } \\
2005\end{array}$ & $\begin{array}{l}119 \text { mulheres } \\
\text { com câncer de } \\
\text { mama }\end{array}$ & $\begin{array}{l}* 30 \text { minutos de } \\
\text { caminhada } \\
\text { intensidade } \\
\text { moderada em casa } \\
\text { de } 6 \text { a } 5 \text { vezes por } \\
\text { semana. }\end{array}$ \\
\hline
\end{tabular}




\begin{tabular}{|c|c|c|}
\hline & & $\begin{array}{l}\text { **Redução da fadiga } \\
\text { muscular. }\end{array}$ \\
\hline $\begin{array}{ll}\text { Fairey et } \\
\text { al, 2005 }\end{array}$ & $\begin{array}{l}53 \text { mulheres } \\
\text { com câncer de } \\
\text { mama }\end{array}$ & $\begin{array}{l}\text { *Exercício com } \\
\text { cicloergômetro } 3 \\
\text { vezes semanais } \\
\text { durante } 15 \text { semanas. } \\
\text { **Aumento da } \\
\text { atividade citotóxica } \\
\text { das células Killer. }\end{array}$ \\
\hline $\begin{array}{l}\text { Courneya } \\
\text { et al, } 2007\end{array}$ & $\begin{array}{l}242 \text { mulheres } \\
\text { com fadiga } \\
\text { muscular } \\
\text { durante a } \\
\text { quimioterapia } \\
\text { no no câncer } \\
\text { de mama }\end{array}$ & $\begin{array}{l}\text { *Exercícios } \\
\text { aeróbicos } \\
\text { e de resistência. } \\
* * \text { Melhora da auto- } \\
\text { estima, aptidão } \\
\text { física e composição } \\
\text { corporal. } \\
\text { Não houve melhora } \\
\text { da fadiga durante a } \\
\text { quimioterapia. }\end{array}$ \\
\hline $\begin{array}{l}\text { Diettrich et } \\
\text { al, } 2007\end{array}$ & 24 mulheres & $\begin{array}{l}* 30 \text { minutos de } \\
\text { esteira ergométrica, } \\
\text { alongamentos de } 5 \\
\text { minutos. } \\
\text { **Redução da fadiga } \\
\text { muscular. }\end{array}$ \\
\hline $\begin{array}{l}\text { Adamsen } \\
\text { et al, } 2009\end{array}$ & $\begin{array}{l}196 \text { mulheres } \\
\text { com fadiga } \\
\text { muscular } \\
\text { durante a } \\
\text { quimioterapia } \\
\text { no câncer de } \\
\text { mama }\end{array}$ & $\begin{array}{l}\text { *Exercícios resistido } \\
\text { de alta e baixa } \\
\text { intensidade, } \\
\text { relaxamento, } \\
\text { conscientização } \\
\text { corporal } \\
\text { massagem. } \\
\text { **Redução da fadiga } \\
\text { muscular, melhora } \\
\text { da capacidade } \\
\text { aeróbica, força } \\
\text { muscular e bem } \\
\text { estar emocional. }\end{array}$ \\
\hline $\begin{array}{l}\text { Haines et } \\
\text { al, } 2010\end{array}$ & $\begin{array}{l}89 \text { mulheres } \\
\text { com câncer de } \\
\text { mama }\end{array}$ & $\begin{array}{l}\text { *Programa de treino } \\
\text { de equilíbrio, } \\
\text { flexibilidade, } \\
\text { coordenação } \\
\text { relaxamento. } \\
* * \text { Melhora da } \\
\text { qualidade de vida. }\end{array}$ \\
\hline $\begin{array}{l}\text { Battaglini } \\
\text { et al., } 2006\end{array}$ & $\begin{array}{lr}20 & \text { mulheres } \\
\text { com fadiga } \\
\text { muscular no } \\
\text { câncer } & \text { de } \\
\text { mama } & \end{array}$ & 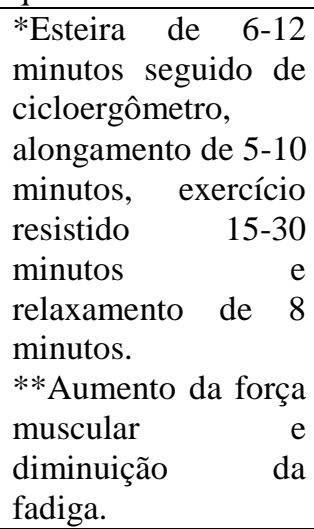 \\
\hline $\begin{array}{l}\text { Dietrrich } \\
\text { et al, } 2006\end{array}$ & $\begin{array}{lr}8 & \text { mulheres } \\
\text { com } & \text { fadiga } \\
\end{array}$ & $\begin{array}{l}\text { *Caminhada de } 12 \\
\text { semanas (programa }\end{array}$ \\
\hline
\end{tabular}

\begin{tabular}{|c|c|c|}
\hline & $\begin{array}{l}\text { muscular } \\
\text { durante a } \\
\text { quimioterapia } \\
\text { no pós- } \\
\text { operatório de } \\
\text { câncer de } \\
\text { mama }\end{array}$ & $\begin{array}{l}\text { de } 5 \text { minutos de } \\
\text { alongamento, } 30 \\
\text { minutos de esteira - } \\
60 \% \text { da FCmáx, } 40 \\
\text { minutos de esteira - } \\
60-80 \% \text { da FCmáx e } \\
4 \quad \text { minutos de } \\
\text { alongamento final. } \\
* * \text { Redução da fadiga } \\
\text { muscular. }\end{array}$ \\
\hline $\begin{array}{l}\text { Noémie et } \\
\text { al, } 2015\end{array}$ & $\begin{array}{l}102 \text { mulheres } \\
\text { com fadiga } \\
\text { muscular } \\
\text { durante a } \\
\text { quimioterapia } \\
\text { no câncer de } \\
\text { mama }\end{array}$ & $\begin{array}{lr}\text { *18 semanas de } \\
\text { exercícios aeróbicos } \\
\text { e resistidos } \\
\text { semanas após o } \\
\text { diagnóstico } & \text { de } \\
\text { câncer de mama } \\
\text { durante } \\
\text { quimioterapia. } \\
\text { **Diminuição da } \\
\text { fadiga muscular } \\
\text { durante o } \\
\text { tratamento, aumento } \\
\text { da aptidão } \\
\text { cardiorrespiratória, } \\
\text { aumento } \\
\text { qualidade de vida e } \\
\text { melhora da força } \\
\text { muscular. }\end{array}$ \\
\hline $\begin{array}{l}\text { Do } \\
\text { Junghwa et } \\
\text { al, } 2015\end{array}$ & $\begin{array}{l}62 \text { mulheres } \\
\text { com fadiga } \\
\text { muscular no } \\
\text { câncer } \\
\text { mama }\end{array}$ & $\begin{array}{l}\text { *Exercícios } \\
\text { aeróbicos, } \\
\text { alongamentos } \\
\text { fortalecimento } \\
\text { muscular durante } 80 \\
\text { minutos, } 5 \text { vezes por } \\
\text { semana com duração } \\
\text { de } 4 \text { semanas. } \\
\text { **Melhora da fadiga } \\
\text { muscular, dor, } \\
\text { náuseas e dispneia. }\end{array}$ \\
\hline
\end{tabular}

Fonte: Levantamento bibliográfico, 1997 à 2015.

*Intervenções aplicadas no câncer de mama.

**Resultados encontrados após as intervenções.

\section{DISCUSSÃO}

\section{A atividade física tem demonstrado} um papel importante no aumento de número e atividade das células NK, durante e imediatamente após vários tipos de exercícios (KENDAL et al,1990). Esse aumento deve-se a secreção de catecolaminas e epinefrina aumentadas durante a realização de 
exercícios. Evidências apontam que um aumento no gasto energético através do exercício físico proporcionaria um impacto positivo na sobrevida e minimizaria a morbidade relacionada ao câncer de mama (HOLMES et al, 2005).

De acordo com Kolden et al. (2002), estudos demonstram os benefícios da prática da atividade física na qualidade de vida relacionada a saúde, com redução da fadiga muscular, diminuição de ansiedade e depressão, melhora da capacidade funcional, controle de peso e menores durações da neutropenia, níveis mais altos de hemoglobina e maior atividade das células NK e por isso tem sido enfatizado em pacientes com câncer ou em tratamento da doença (COURNEYA et al, 2004). Os exercícios trazem possíveis efeitos benéficos em relação a imunoestimulação (GLEESON et al, 2011).

Os exercícios físicos podem promover alterações positivas nos parâmetros imunológicos. Realizando exercícios aeróbicos (ciclismo, corrida e natação) e exercícios de resistência (aumento da força muscular, com exercícios isométricos, isotônicos ou isocinético), foi possível verificar que o número de leucócitos, linfócitos e neutrófilos aumentaram. Também pode-se observar um aumento da resistência do organismo frente citoxicidade, havendo após o exercício uma estabilização no número de células NK e linfócitos T (SAWADA et al, 2010).
Kelm et al. (2000), ao investigar o efeito de um programa de treze semanas de treinamento de força muscular e exercício aeróbico, que foi realizado duas vezes por semana em pacientes submetidos a quimioterapia, pode observar um aumento do número de células NK. Apesar das alterações imunológicas serem eventualmente de curta duração, mudanças podem se acumular ao longo do tempo (TIMMONS, 2007) sendo importante para orientar a ideal dose de exercício, já que seu principal objetivo com pacientes com câncer é suprimir a inflamação em casos de elevação (PLOEGER et al, 2009) e aumentar a vigilância imunológica (FAIREY et al, 2002)

Acredita-se que exercícios exaustivos possam levar a um quadro imuno-supressor transitório, aumentando a susceptibilidade às infecções (PEDERSEN et al, 2000). Explicações da neutropenia podem estar relacionados à receptores da membrana celular, iniciando uma cascata de eventos intracelulares levando ao lançamento de enzimas e à produção de reativos intermediários de oxigênio, ocasionado um extravasamento de neutrófilos aos tecidos lesados (NIEMAN et al, 1994).

Já os exercícios de intensidade moderada, praticadas regularmente, mostram uma associação na diminuição das infecções, principalmente relacionada com o trato respiratório superior (NIEMAN et al, 1994). 
Saxton et al. (2003), avaliaram o exercício aeróbico com exercício de força excêntrica. Pode-se observar aumentos na contagem dos leucócitos, principalmente os neutrófilos. O recrutamento dos leucócitos pode ser decorrente de efeitos dos exercícios aeróbicos, decorrente dos efeitos das catecolaminas e do cortisol e IL-6 na mobilização celular dos órgãos linfoides (RAMEL， 2003; SIMONSON， 2004; FOSCHINI, 2007). Os exercícios de força estão associados com uma resposta local, já os exercícios aeróbicos com uma resposta imune sistêmica (SAXTON, 2003; TIMMONS, 2007).

A prática de exercícios físicos como programas de caminhada pode ser benéfica a pacientes oncológicos, incluindo aumento da energia física e/ou da capacidade funcional e também da qualidade de vida, além de outros benefícios psicológicos como aumento no senso de confiança e habilidade para desafiar o câncer e seu tratamento, além de poder minimizar a fadiga muscular e conservar a capacidade funcional (COURNEYA et al, 2007).

\section{CONCLUSÃO}

Os estudos demonstraram a importância dos exercícios físicos durante o tratamento quimioterápico no câncer de mama, ressaltando os exercícios de força muscular e aeróbicos, como caminhada, esteira, bicicleta ergométrica na melhora de qualidade de vida, na fadiga muscular e na mielodepressão.

\section{REFERÊNCIAS}

1. ADAMSEN, L. et al. Effect of a multimodal high intensity exercise intervention in cancer patients undergoing chemotherapy: Randomized controlled trial: British Medical Journal, London; v.13, no 339, p. b3410, 2009.

\section{BATTAGLINI, C. et al. Efeitos do} treinamento de resistência na força muscular e níveis de fadiga em pacientes com câncer de mama. Revista Brasileira de Medicina do Esporte, São Paulo; v.12, n.3, p. 153-158, 2006.

3. BRASIL. Ministério da Saúde. Secretaria Nacional de Assistência à Saúde. Instituto Nacional de Câncer. Estimativas da incidência e mortalidade por câncer no Brasil. Rio de Janeiro: INCA, 2015.

4. COURNEYA, KS, Mackey JR, Bell GJ, Jones LW, Field CJ, Fairey AS. Randomized Controlled Trial of Exercise Training in Postmenopausal Breast Cancer Survivors: Cardiopulmonary and Quality of Life Outcomes. J Clin Oncol; 21:1660-8, 2003.

5. COURNEYA, KS, Segal RJ, Mackey JR, Gelmon K, Reid RD, Friedenreich CM, et al. Effects of aerobic and resistance exercise in breast cancer patients receiving 18 Exercícios em Pacientes Oncológicos: Reabilitação Projeto Diretrizes Associação Médica Brasileira adjuvant chemotherapy: a multicenter randomized controlled trial. J Clin Oncol; 25:4396-404, 2007.

6. COURNEYA, KS, Mackey JR, Quinney HA. Neoplasias. In: Pesquisas do ACSM para fisiologia do exercício clinico: afecções musculoesqueléticas, neuromusculares, 
neoplásicas, imunológicas e hematológicas. Rio de Janeiro: Guanabara-Koogan, 2004.

7. DIETTRICH, SHC; Miranda CRR; Honer, MR.; Furtado, ER.; Corrêa Filho, RAC. Efeitos de um programa de caminhada sobre os níveis de fadiga em pacientes com câncer de mama. R. bras. Ci e Mov; 14(1): 7-12, 2006.

8. DIETTRICH, SHC, Eficácia de um programa de atividade Física nos níveis de fadiga em pacientes em tratamento de câncer de mama. Campo Grande: [ tese de doutorado- Faculdade Estácio de Sá], 2007.

9. DO Junghwa, Youngki Cho, Jaeyong. Effects of a 4-Week multimodal rehabilitation program on quality of life, cardiopulmonary function, and fatigue in breast cancer patients. J Breast Cancer. Mar; 18(1): 87-96, 2015.

10. FAREY AS. et al. Randomized controlled trial of exercise and blood immune function in postmenopausal breast cancer survivors. Journal of Applied Physiology, Bethesda; v. 98, no 4, p. 15341540, 2005.

11. FAIREY, AS, Courneya KS, Field CJ and Mackey JR. Physical exercise and immune system function in cancer survivors: a comprehensive review and future directions. Cancer; 94: 539-551, 2002.

12. FOSCHINI, D, Prestes J. Respostas hormonais e imunes agudas decorrentes do treinamento de força em Bi-Set. Fit Perf J.; 6(1): 37-44, 2007

\section{GLEESON, M, Bishop NC, Stensel DJ,} Lindley MR, Mastana SS and Nimmo MA.

The anti-inflammatory effects of exercise: mechanisms and implications for the prevention and treatment of disease. Nat Rev Immunol, 2011.
14. HAINES TP, Sinnamon P, Wetzig NG, Lehman M, Walpole E, Pratt T, et al.

Multimodal exercise improves quality of life of women being treated for breast cancer, but at what cost? Randomized trial with economic evaluation. Breast cancer Res Treat; 124:163-75, 2010.

15. HAWKE TJ. Muscle stem cell and exercise training. Exerc sport sci Rev; 32(2): 63-68, 2005.

16. HOLMES, MD, Chen Wye, Feskanich D, Kroenke $\mathrm{CH}$, Colditz GA. Physical activity and survival after breast cancer diagnosis. Jama; 39(20):2479-86, 2005.

17. KENDALL, A, Hoffman-Goetz L, Houston M. Exercise and blood lymphocyte subset responses: intensity, duration, and subjects fitness effects. J Appl Physiol; 69: 251-60, 1990.

18. KELM, J. et al. Auswirkungen eines kraft-und ausdaerorientierten traininh gs wahred regionater chemotherapie bei metartasierendem rectum carcinoma. Fall studiels beitragzur chururgirchen Onckologie, Der Cherurg; v.71, p. 944-948, 2000 .

19. KOLDEN, GG, Strauman TJ, Ward A, Kuta J, Woods TE, Schneider KL, et al. A pilot study of group exercise training (GET) for women with primary breast cancer: feasibility and health benefits. Psycho-Oncology; 11: 447-56, 2002.

20. MOCK, V, Frangakis C, Davidson NE, Ropka ME, Pickett M, Poniatowski B, et al. Exercise manages fatigue during breast cancer treatment: a randomized controlled trial. Psychooncology;14:464-77, 2005.

21. MOCK, V, Pickett M, Ropka ME, Lin EM, Stewart KJ, Rhoder VA, Mcclarke R. Fatigue and quality of life: outcomes of exercise during cancer treatment. Cancer Practice; 9:119-127, 2001. 
22. MOCK, V, Dow KH, Meares CJ, et al. Effects of exercise on fatigue, physical functioning and emotional distress during radiation therapy for breast câncer. Oncol Nurs Forum; 24:991-1000, 1997.

23. NIEMAN, DC, Nehlsen-Cannarella SL. The imune response to exercise. Semin Hematol; 31: 166-79, 1994.

24. NOÉMIE, T, et al. Effects of an 18-week exercise programme started early during breast cancer treatment: a randomized controlled trial. BMC Med; 13: 121, 2015.

25. PEDERSEN, BK, Hoffman-Goetz L. Exercise and the imune system: regulation, integration, and adaptation. Physiol Rev; 80(3): 1055-1081, 2000.

26. PLOEGER, HE, Takken $T$, De Greef MH and Timmons BW. The effects of acute and chronic exercise on inflammatory markers in children and adults with a chronic inflammatory disease: a systematic review. Exerc Immunol Rev; 15: 6-41, 2009.

27. RAMEL, A, Wagner KH, Elmadfa I. Acute impacto f submaximal resistance exercise on immunological and hormaonal parameters in Young men. J Sports Sci. 2003; 21(12): 1001-1008.

28. RICHARDSON, A, Ream E, WilsonBarnett $\mathrm{J}$. Fatigue in patients reciving chemotherapy: Patterns of changes. Cancer Nursing; 21: 17-30, 1998.

29. SCHWENKGLENKS, M. et al. Neutropenic event risk and impaired chemotherapy delivery in six European audits of breast cancer treatment. Supportive care in cancer, Berlin; v. 14, p. 901-909, 2006.

30. SAXTON, JM, Claxton D, Winter E, Pockeley G. Peripheral blood leucocyte functional responses to acute eccentric exercise in humans are influenced by systemic stress, but not by exercise-induced muscle damage. Clin Sci (Lond); 104(1); 6977, 2003

31. SAWADA, S, Kobayashi K, Ohtani M and Fukusaki C. Cystine and theanine supplementation restores high-intensity resistance exercise-induced attenuation of natural killer cell activity in well-trained men. J Strength Cond Res; 24: 846-851, 2010.

32. SIMONSON, SR, Jackson CGR. Leukocytosis occur in response to resistance exercise in men. J Strength Cond Res; 18(2): 266-271, 2004.

33. SKALA, KA, Lacasse C. Patient education for fatigue. Oncol Nursing Foram; 19: 1537-1539, 1992.

34. THORSEN, L, Skovlund E, Strømme SB, Hornslien K, Dahl AA, Fosså SD. Effectiveness of physical activity on cardiorespiratory fitness and healthrelated quality of life in young and middleaged cancer patients shortly after chemotherapy J Clin Oncol; 23:2378-88, 2005.

35. TIMMONS, BW. Exercise and immune function in children. Am J Lifestyle Med; 1: 59-66, 2007. 